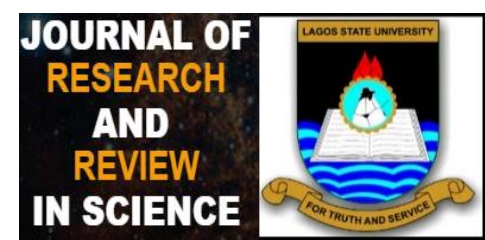

ORIGINAL REASEARCH

\title{
API Assisted Semantic Matching for Subjective Online Examination System
}

\section{Oluwatoyin Enikuomehin and Uthman Dosumu}

Department of Computer Science, Faculty of

Science, Lagos State University, Nigeria

\section{Correspondence}

Oluwatoyin Ayokunle Enikuomehin, Department of Computer Science, Faculty of Science, Lagos State University, Nigeria. Email:toyinenikuomehin@gmail.com

\begin{abstract}
:
Introduction: Online examination systems exist as an attempt to expand the frontiers of learning and testing. This development has been limited by the strictness of the examination format vis-à-vis keyword match which are boundary-defined. This constraint has led to poor adoption of online testing systems which are essential in handling the problem associated with large enrolment. Existing systems do not allow examiners to test for knowledge in ways that they seem due; similar to the conventional classroom testing system.

Aim: To develop an Application Programming Interface (API) assisted semantic matching for subjective online examination system. The proposed system will take advantages of web resources to reduce time, cost and other constraints associated with location defined examination.

Materials and Methods:We leveraged on the advances in Natural Language Processing and the success emerging from same and therefore remodelled the examination system against some known concepts in text summarization, term dependencies, semantic tagging and corporal buildup using a standalone global API for semantic interpretation of answers.

Results: We improve the Levenshtein distance between two strings $a, b$ using the triangular inequality to identify the relationship of two terms as applicable between questions and answers. The metric was coded in php with a MySQL supporting backend. The Levenshtein distance was denoted in php with the int levenshtein ( string \$str1, string \$str2).The complexity of the algorithm is $O\left(m^{*} n\right)$, where $n$ and $m$ are the length of str1 and str2.

Conclusion: The output of the research is a subjective examination system that allows the self-grading of essay type question using a web based semantic API.

Keywords: Semantic API, Levenshtein distance, Subjective Examination, Subject online examination, Question Answering Systems
\end{abstract}




\section{INTRODUCTION}

The keen seek for knowledge has been existing as early as humanity itself. This led to the immediate adoption of Natural Language as a means of processing the human need with the Computer. Literature also agrees that Natural Language has been a major tool for Question Answering Systems (QAS), a "middle-man" between Information Retrieval and Natural Language Processing. QAS is a microcosm of the information seeking task. Examinations are forms of Question Answering systems. Online examination systems exist as an attempt to expand the frontiers of learning and testing. The system secondarily aims at taking the advantages of web resources to reduce time, cost and other constraint associated with location defined examination. They have never been aimed as a complete replacement. The development has been limited by the strictness of the examination format visà-vis keyword match.

Online examination system exist as an attempt to remove the existing flaws in the manual system of conducting examinations [1]. These flaws are of varied dimension and with the polynomial increasing enrollment in Universities, the need for an improved system that reduces the identified flaws and increase productivity cannot be over emphasized. This research reports a developed platform for examination conduct with the conventional grading system of strict keyword match. The research identifies the reason for low subscription to the generalized online and web based examinations and used evolving scientific concepts to improve the convention by adding a semantic analyzer of students' feedback based on the use of a real time meaning generation algorithm. [2][3][4] Concluded that online examinations are convenient, and fast however the rate of adoption is low due to its strictness in implementation. Given the answer to a question to be "go", the system only identifies "go" as the only correct answer where other synonyms such as "proceed" among others would be marked wrong. We appreciate the effort on the research on term matching in the Natural Langauage Processing domain and the success emerging from same, and therefore remodel the examination system against some known concepts in text summarization, term dependencies, semantic tagging and corporal build-up for an improved online grading system. Our approach will give the user a leverage to set questions in his/her own way especially the process of implementing subjective testing. Generally, online examination is aimed at the following objectives:

- To allow test-takers, at different places, to take the test at the same time through the online examination platform.

- To reduce time taken for examination grading and score generation.

- To build a question repository.
Several online examination systems have been developed in the last decade and most, targeted at solving the specific problem of a well-defined set of users, however all of the non-theoretical developed solutions have limited their question base to multiple choice type questions (MCQ) where the test takers are given a question with a range of answer and expected to choose one which matches the answer contained at the backend. A typical MCQ is as follows:

Question: Bread is made from one of the following: a. Flour b. Stone c. lime stone d. Alkaline

At the backend of the application, the answer will be stored as "a". Students are expected to select option a for the correct answer. Few examiners also use the TRUE or FALSE type, as exemplified below: Trump is the name of the US president

\section{TRUE FALSE}

In this case, we can note that the question still exist a one word answer of either TRUE or FALSE. These methods are called objective examination system. More often, examiners have debated the true reflection of the objective based examination as a true test of knowledge and have always felt that the system low level of adoption can been attributed to the impossibility of allowing students to answer conventional questions as they could have done in the physical examination halls. This paper presents a novel solution to this concern that implements the Subjective examination system. The closest subjective examination system that ever existed only allow a one-word answer unlike the findings reported in this paper that allows for essay type questions. Students can be asked:

\section{Question: What is a noun?}

Answer: A noun is a name of any person Animal, Place or Thing.

The subjective examination is achieved by breaking the user supplied answer into lexically related tokens and perform semantic matching using appropriate Part Of Speech (POS) algorithm.

\section{MATERIAL AND METHODS}

Part of Speech algorithm were used for term identification before the leventein distance algorithm coded in php was invoked into find the closeness of terms. This precedes the implementation of semantic algorithm embedded in the WordNet API such that for any answer, the semantic similarity is first determined by the system. The interactive components of the new system is hereby presented. [5] compared several software used for online surveying, examination etc and submits that the need for an improved system cannot be over emphasized.

It will help organizations solve the present challenges they are facing in attracting eligible and professional employees by providing a software system that processes the application of different applicants [6]. It will also save them time and cost of recruiting. 


\subsection{ONLINE SUBJECTIVE EXAMINATION}

[7] assessed the managerial implication of the computer based online business education. They discovered that the skills of the student in using computer is crucial in the successful running of online business education. [8] discovered that assessing the managers up to middle level management staff remained smooth through online objective type questions, as these managers are mostly concerned about tactical and operational details only.

However, online subjective assessment approach remained useful to the senior managers that are involved into policy formation and written correspondence. The study perceives that as the managers rise from middle management cadre to strategic management level, their job becomes more operational and strategic to the point that require more subjective expression [9], which supports the findings of [8].

\subsubsection{Subjective Test Algorithm}

Subjective questions and the required answers are presented in natural language, however the ambiguity contained natural language permits users to express the same meaning in different ways. Accurate ratings of subjective questions are almost impossible just as objective questions. The systems will conventionally analyze the supplied answers and then use appropriate matching algorithm to determine relevance. The match rating Approach (MRA) is a phonetic algorithm which was developed by Western Airlines in 1977 for indexing and comparing homophonous names. MRA uses distance calculation between two words. The distance approach was studied and improved upon using a standard edit distance algorithm to compare similarity of two terms.

\subsection{PROPOSED SYSTEM}

The Levenshtein edit distance approach is considered in this paper. TheLevenshtein distance is an edit distance implementation of the Wagner Fischer Algorithm. It is a string metric for measuring the difference between two sequences. Informally, the Levenshtein distance between two words is the minimum number of single-character edits (i.e. insertions, deletions or substitutions) required to change one word into the other. It is named after Vladimir Levenshtein, who considered this distance in 1965. It may also denote a larger family of distance metrics which is related to pairwise string alignments[10].

Mathematically, the Levenshtein distance between two strings $a, b$ (of length $|a|$ and $|b|$ respectively) is given by lev $a, b(|a|,|b|)$ Where

$$
\operatorname{lev}_{a, b}(i, j)= \begin{cases}\max (i, j) & \text { if } \min (i, j)=0, \\ \min \begin{cases}\operatorname{lev}_{a, b}(i-1, j)+1 \\ \operatorname{lev}_{a, b}(i, j-1)+1 \\ \operatorname{lev}_{a, b}(i-1, j-1)+1_{\left(a_{i} \neq b_{j}\right)}\end{cases} & \text { otherwise. }\end{cases}
$$

Figure 1.0: Levensthtein distance measure.

For the sake of the development of this software, the above was coded in php for easy of web integration as the application is web based.

int levenshtein ( string \$str1, string \$str2 )

int levenshtein ( string \$str1, string \$str2, int \$cost_in $s$, int \$cost_rep, int \$cost_del )

\subsubsection{Answer Processing}

This process of breaking user supplied answers into distinct word and comparing the standard keyword to user supplied answer is the most crucial part of the subjective examination process. Along with the standard keywords, the synonyms of the keywords submitted by the user will also be extracted and tokenized using the Porters Algorithm [11]. The tokenized keywords are the words that provide the key concept which are of significance in a document. The keywords that get repeated very often in a document are given less importance in semantic ranking so as to preserve the meaning. But the keywords which occur rarely in a document will have great importance. The synonyms of the keywords will be extracted from a lexical resource; Wordnet. Once an answer has been identified, the shallow parsing using [12] is leveraged upon to extract only the relevant word or phrase in answer to the question.

\subsubsection{Answer Structures}

\subsubsection{One-line answer}

One-line answer or Define type questions may have a sentence which may have many words. Therefore, we cannot fix the number of words. For the purpose of this work, we used the full stop sign to denote the end of the supplied answer as a sentence may contain many words.

\subsubsection{One-line answer}

Same case applies to Answer the question in "details" or "briefly" type questions. We cannot fix the size of paragraphs or pages. Generally, a restriction of words will be provided such that every answer has to be in a given minimum length, this is done to avoid the case of awarding full mark to users that supply the system with only the system standard keyword. i.e. user can submit an answer of 20 words or more than, if 20 words is the minimum length of words required. This implies that less than 20 words answers will not be considered as desirable answer. 


\subsubsection{Weighting user supplied answer}

Weighting is used to grade the correctness of the answer supplied by the use. It contains the following constituents:

Keywords

The keywords of the model answer will be converted into lowercase letters before it is then stored in the database. These keywords are extracted by our system and stored in a multidimensional array. These words are checked in the student's answer and the percentage of occurrence is noted and further used in awarding marks for the supplied answers. The operation identifies the keyword in the answers and are expected to match up in meaning to the contextual keywords provided in the database which are then used for similarity comparison. Grades are awarded after the application of the semantic analysis implemented by the wagnerfischer algorithm of the leventien distance model.

\section{Synonyms}

Synonyms are required in order to ensure that the supplied answers with same meaning are also marked as correct. This is one of the new findings reported in this paper which includes the use of an API on Wordnet to identify the similarity of the sentences as a mapping to the contained keywords.

Length

Length of the answer is also considered in the system as very important because the student may write the short answer with all keywords included however such answer will loose mark assigned to length as for every answer, a minimum length of answer is required.

\section{RESULTS}

All the above stated procedures were used in the development of a stand alone web based subjective examination software with the several features. The developed system is divided into four distinct modules:

1. Candidate/Student module.

2. Facilitator module.

3. Administrator module.

4. Super-administrator module

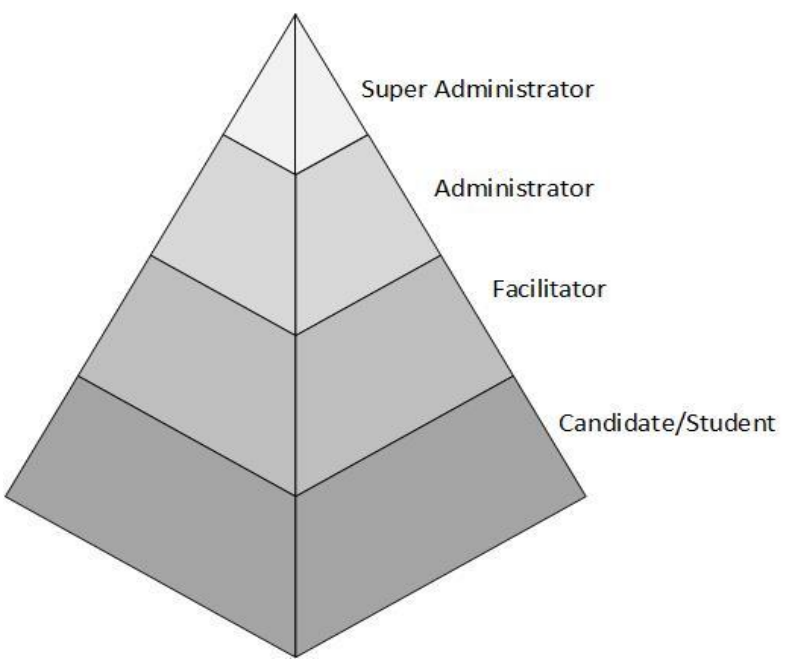

Figure 1: The system modules.

Candidate module: The candidate will logon to the system and take his/her examination. The candidate will get result almost immediately after the completion of the examination.

Facilitator module: The facilitator activates the examination including date and time.

Administrator module: The administrator is in charge of uploading questions to the question bank, deciding the pass mark for the exam and also activating a facilitator. Super-administrator module: The super-administrator seats at the highest level of the system hierarchy, overseeing all user activities. Only the superadministrator has the privilege to activate the Administrator.

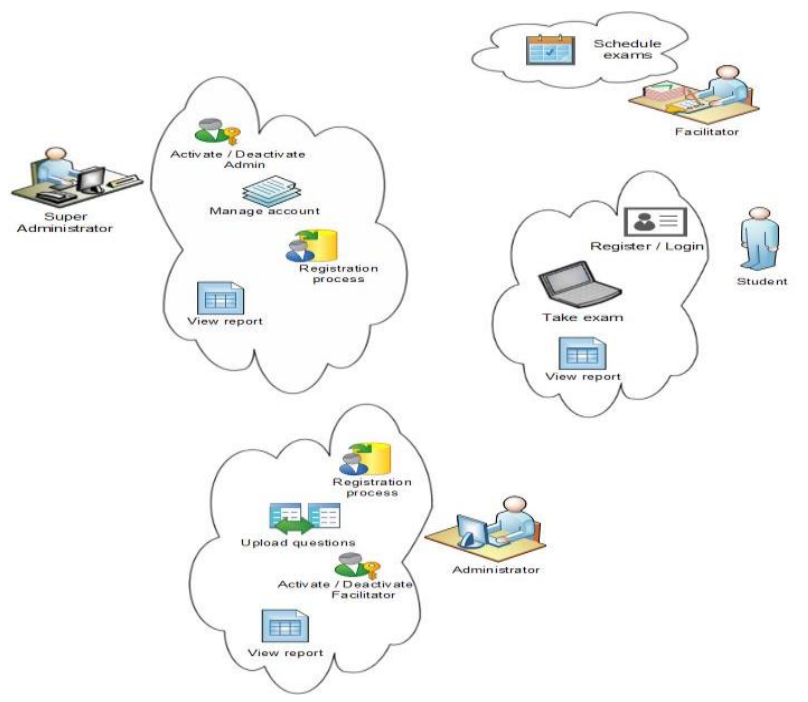

Figure 2: The Ecosystem of the Subjective
examination system

Super-administrator

- The administrator has the full-fledged rights over the Online Examination System.

- Activation and deactivation of an Administrator

- Can add/delete an administrator or facilitator.

Administrator

- Activation and deactivation of a facilitator

- Can view the accounts.

- Can change the password. 
- Add/delete/edit question in the question bank.

- Can access the accounts of the faculty Facilitator members/students.

- $\quad$ Aid the exam by providing the date and time of the exam and also activating the time provided for student to spend on a question.

Student

- Can change password.

- Can view the different categories of examination available in the exam scheduler.

- Can print result

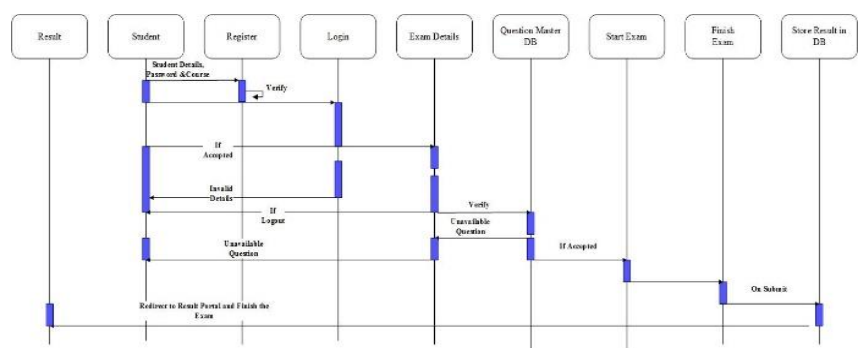

Figure 3: Sequence Diagram Model of how student take an exam and give the result.

\section{DISCUSSION}

User needs to register as a student before being granted the access to log into the system and save student details in the database. The logon process follows immediately. The login describes the interaction between the student as an object and the login, if on logging in the student enters an invalid details the system prompts an error message and direct the user back to the login page else the system refers user to the examination details page. The examination details object displays the user and the registered details (username, reference number, number of questions available for the exam as well as the type of examination etc.) which include a start button that links the Examination details with the Start examination page. If such examination does not exist, the system displays a "No Exam" message and direct the user back to the login page. The examination questions are stored in the Question Master Database. If questions are available for the examination, the system fetches and display the questions stored in the question bank where the course exist. Student interacts with the finish examination button when the student finishes the examination. The system stores and compare the user submitted answers with the correct answers stored in the database using the earlier defined procedure and grades the student appropriately. Scores are automatically activated on the students' result page. Finally, the result page interacts with the system database to fetch the result of the student and display.

\section{CONCLUSION}

This paper presents the output of a research solving the problems associated with objective online examination. The paper reports a novel approach to subjective online examination using a global API for semantic interpretation. The analysis time is improved upon by first using the Levenshtein distance between two strings $\mathrm{m}, \mathrm{n}$ to model the similarity of terms.

The solution was developed using PHP, JavaScript and MySQL as the major programming languages and database used for developing the entire system. One constraint observed but have not been looked into as it falls out of the scope of this paper, is the effect of speed typing on subjective examination. Such may require the need to carry out an on the spot experimentation. We recommend the use of this system as the enrolment rate has now increased nationwide.

\section{ACKNOWLEDGEMENTS}

We would like to acknowledge the contributions of Prof. B.S. Aribisala, Chairman, ICT Committee of the Lagos State University, Nigeria for his productive criticism which has led to the improvements reported in this paper. We would also like to show our gratitude to the organizers of the 2017 Science conference where this work was first showcased and feedbacks from participants were accommodated as appropriate. We immensely thank members of the department of computer science, Lagos State University, Nigeria fo their time to test the developed solution.

\section{COMPETING INTERESTS}

Authors have declared that no competing interests exist.

\section{AUTHORS' CONTRIBUTIONS}

Dr. Oluwatoyin Enikuomehin conceived the presented idea, designed the model and the computational framework to which this paper is based. He did the experimentation and encouraged $\mathrm{mr}$. Uthman Dosumu to carry out the implementation via PHP/MYSQL. Dr. Oluwatoyin Enikuomehin wrote the manuscript with contribution from the other author. All authors discussed the output and contributed to the final manuscript.

\section{REFERENCES}

1. Shen, J., Cheng, K. E., Bieber, M., \& Hiltz, S. R. (2004). Traditional in-class Examination vs. collaborative online examination in asynchronous learning networks: Field evaluation results. AMCIS 2004 Proceedings, 364.

2. Zhang, L., Zhuang, Y. T., Yuan, Z. M., \& Zhan, G. H. (2006, July). A web-based examination and evaluation system for computer education. In Advanced Learning Technologies, 2006. Sixth International Conference on (pp. 120124). IEEE.

3. Ullah, A., Xiao, H., \& Lilley, M. (2012, June). Profile based student authentication in online examination. In Information Society (i-Society), 
2012 International Conference on (pp. 109113). IEEE.

4. Chellappa, R. K., \& Sin, R. G. (2005). Personalization versus privacy: An empirical examination of the online consumer's dilemma. Information technology and management, 6(2), 181-202.

5. Zhou, Z. Q., Xiang, S., \& Chen, T. Y. (2016). Metamorphic testing for software quality assessment: A study of search engines. IEEE Transactions on Software Engineering, 42(3), 264-284.

6. He, W. (2013). Examining students' online interaction in a live video streaming environment using data mining and text mining. Computers in Human Behavior, 29(1), 90-102.

7. Fontenot, R. J., Mathisen, R. E., Carley, S. S., \& Stuart, R. S. (2015). Predictors of Enrolling in Online Courses: An Exploratory Study of Students in Undergraduate Marketing Courses. Journal of Educators Online, 12(1), $\mathrm{n} 1$.

8. Smith, A. A., Smith, A. D., \& Rupp, W. T. (2014). Strategic insights into the critical success factors online for-profit educational providers: case study. International Journal of Business Information Systems, 17(1), 1-32.

9. Devi, M. S., \& Mittal, H. (2016). Machine Learning Techniques with Ontology for Subjective Answer Evaluation. arXiv preprint arXiv:1605.02442.

10. Ho, T., Oh, S. R., \& Kim, H. (2017). A parallel approximate string matching under Levenshtein distance on graphics processing units using warp-shuffle operations. PloS one, 12(10), e0186251.

11. Pande, B. P., Tamta, P., \&Dhami, H. S. (2015). A simple algorithm for the problem of suffix stripping. International Journal of Applied Linguistics, 25(3), 315-328.

12. Liu, S., Zhang, Z., \& Liu, P. (2015, May). Lexicalized Token Subcategory and Complex Context Based Shallow Parsing. In Workshop on Chinese Lexical Semantics (pp. 465-470). Springer, Cham. 